\title{
Modellierung und Simulation von Vollmantel- zentrifugen als ein Aspekt der voranschreitenden Digitalisierung in der Fest/Flüssig-Trennung
}

\author{
Simon Hammerich*, Marco Gleiß* und Hermann Nirschl
}

\begin{abstract}
Im Zuge der Initiative Industrie 4.0 erhöht sich der Digitalisierungsgrad auch in der Prozessindustrie. Eine enge Verknüp fung von Analyseverfahren und dynamischer Prozessmodelle erlaubt die Optimierung der Prozesskontrolle und Steuerung auch in der industriellen Trenntechnik. Zu diesem Zweck müssen mathematische Modelle zur Beschreibung der Prozess ebene in Echtzeit bekannt sein. Im Bereich der Fest/Flüssig Trennung erzwingt die voranschreitende Digitalisierung die Entwicklung von numerischen Methoden und von Short cut Modellen zur Vertiefung des Prozessverständnisses und der besseren Auslegung der Anlagen. Aufgelöste Strömungssimulationen dienen hier als Unterstützung zu Experimenten und zur Ableitung geeigneter Short cut Modelle. Am Beispiel von Vollmantelzentrifugen wird die Entwicklung dynamischer Prozessmodelle und deren Anwendung beschrieben.
\end{abstract}

Schlagwörter: Digitalisierung, Dynamische Prozessmodelle, Numerische Strömungssimulationen, Prozesssteuerung, Zentrifugation

\section{Modeling and Simulation of Solid-Bowl Centrifuges as an Aspect of the Advancing Digitization in Solid-Liquid Separation}

\begin{abstract}
As a part of the initiative Industry 4.0, the level of digitization in the process industry is increasing. Through a close link between analytics and dynamic modeling, digitization enables the optimization in processing industry or the model based control. For this purpose, mathematical models of the process level enabling real time simulations have to be known. In the field of solid liquid separation the advancing digitization forces the development of numerical flow simulation meth ods and short cut models to deepen process understanding and mathematical process modeling. Resolved flow simula tions should be seen as support for experiments to develop suitable dynamic models for short cut models. Based on the prerequisites, the development of dynamic process models and their application using the example of solid bowl centrifu ges is described.
\end{abstract}

Keywords: Centrifugation, Digitization, Dynamic process models, Numerical flow simulation, Process control

\section{Einleitung}

Die derzeit in der Industrie stark voranschreitende Digitali sierung im Rahmen der Initiative Industrie 4.0 hat starke Auswirkungen auf die Kernprozesse „Supply Chain“ inklu sive Produktion, „Asset Lifecycle“ sowie kundenorientierte, datengetriebene Geschäftsmodelle [1]. Treiber dieser Ent wicklung war bisher die Fertigungstechnik, wobei in der Zwischenzeit die Prozessindustrie die Chancen und auch die Risiken (u. a. die Datensicherheit) erkannt hat. Die Digi talisierung der Prozesse zur Partikelproduktion ist sehr viel schichtig, wobei ein wesentlicher Aspekt die besonders von den Universitäten und Forschungseinrichtungen vorange triebene Simulation mit der zugehörigen Erfassung der Pro dukteigenschaften umfasst. Eine der Säulen ist eine umfang reiche Simulation der Prozesse auf unterschiedlichen Zeit und Längenskalen. Sicherlich sind nicht für jeden Prozess alle Skalen und Ebenen abzubilden, alleine jedoch die Ent wicklung von Short cut Modellen zur schnellen Simulation

Simon Hammerich, Marco Gleiß, Prof. Dr. Ing. Hermann Nirschl simon.hammerich@kit.edu, marco.gleiss@kit.edu Karlsruher Institut fur Technologie (KIT), Institut fur Mechanische Verfahrenstechnik und Mechanik, Straße am Forum 8, 76131 Karlsruhe, Deutschland. 
im Wechselspiel mit der Automatisierungstechnik ist ein bislang nicht intensiv verfolgtes Forschungsfeld.

Die derzeit zur Verfügung stehenden Simulationstools sind relativ fokussiert und betrachten einen Prozess nicht in seiner gesamten Breite, sondern zum einen entlang der Pro zesskette und zum andern in seiner verfahrenstechnischen Tiefe im Hinblick auf die Reaktionstechnik oder den Ener gie und Rohstoffeinsatz. Es ist daher denkbar, mittels eines zu entwickelnden Datenmodells die verschiedenen Simula tionstools zu koppeln und einen Datenaustausch sicher zustellen [2]. Dies ist für den Prozessbetrieb in Wechsel wirkung mit der Automatisierungstechnik sinnvoll und erlaubt die prädiktive Prozessentwicklung. Dadurch können unvorhergesehene Ereignisse, unzureichende Prozesssteue rungen und somit Fehlchargen in der Prozessindustrie ver mieden werden. Für eine industrienahe Simulation stellen sich die folgenden Herausforderungen:

Eine ganzheitliche Simulation entlang der kompletten Prozesskette ist für die Verfolgung der Produktentste hung von Vorteil. Dies beinhaltet auch die Einbindung von Energie und Rohstoffeffizienz, Kosten, Lebenszyklus sowie die dazugehörigen Optimierungstools [3].

Die Kopplung von Simulationsverfahren (Fluid, Struk tur, Partikel, Molekularsimulation etc.) auf mehreren Ebenen erscheint ein möglicher Weg zur ganzheitlichen Betrachtung partikulärer Prozesse [2]; allerdings immer im Hinblick auf eine Echtzeit Simulation mit Short cut Modellen mit dem nur unbedingt notwendigen Detailie rungsgrad.

Prädiktive Prozesssimulationen sind derzeit nicht mö glich, würden jedoch eine vorausschauende Steuerung und Regelung erlauben.

Aufgrund der komplexen Materialparameter ist ein klas sischer Scale up anhand der üblichen auf Kennzahlen basierenden Methoden nicht mehr zielführend [4]. Pro zess Simulationen auf der Makroskala, in denen auch das komplexe Materialverhalten abgebildet ist, sind dafür jedoch ein nicht mehr zu vernachlässigendes Werkzeug [5].

Big Data ist ein weiteres in der Industrie 4.0 häufig benutztes Schlagwort. Die Abstraktion und Nutzbar machung der für die Prozesssteuerung oder für die Pro duktqualität unbedingt notwendigen Daten ist ein lohn endes Forschungsziel.

Die Mathematik stellt umfangreiche Optimierungsalgo rithmen, wie das Quasi Newton oder das SQP Verfahren, zur Verfügung [6]. Diese Methoden sind bei den Prozess entwicklern nur bedingt bekannt und finden daher wenig Anwendung. Eine Einbindung von derartigen Tools zur Optimierung der Prozesse auf den verschiedenen Ebenen ist eine lohnende zukünftige Forschungsaufgabe.

Nicht zuletzt ist die Entwicklung von Ausbildungs und Trainingstools ein wichtiger Baustein. In der Prozess industrie eingesetzte Anlagen werden häufig im $24 \mathrm{~h}$ Betrieb gefahren. Eine Ausbildung für Prozessverant wortliche und ein Training für das Studium von unge wöhnlichen Betriebszuständen ist an realen Prozessen nicht realisierbar [7].

Im Gegensatz zur Fertigungstechnik ist für die Prozess industrie die Gewinnung von Messdaten zur Charakterisie rung der Produkteigenschaften von entscheidender Bedeu tung. Für die Simulation sind in der Partikeltechnik physikalische Parameter wie die Partikelform, die Partikel größe, die Agglomeratstruktur, die Wechselwirkungen zwi schen den Partikeln, die Grenzflächeneigenschaften oder das Fließverhalten zu charakterisieren. Diese Parameter be stimmen das Verhalten des gesamten Materials als Kollektiv, was bisher in Simulationen nur sehr schwer oder gar nicht berücksichtigt wird. Eine Verbindung dieser partikelcharak terisierenden Parameter mit anwendungstechnischen Ei genschaften (z. B. Rollwiderstände, Härte und Festigkeiten von Werkstoffen) wäre ein wichtiger Forschungsgegenstand. Scale up Ansätze scheitern im Feststoffbereich, da sich bei spielsweise die Feststoffförderung innerhalb von Trenn aggregaten nicht vorhersagen lässt [8]. Weiterhin können In situ Messungen zur Ermittlung von Partikelformen und Agglomeratstrukturen Vorhersagen hinsichtlich einer Klas sierung oder Abscheidung erleichtern.

Im Hinblick auf eine flexible, robuste Prozessgestaltung sind somit Online oder besser noch In situ Messverfahren notwendig, die als Schnittstelle zur Simulation dienen. Daraus lassen sich Daten in Echtzeit zur Verfügung stellen, die wiederum als Ist Werte für die übergeordnete Validierung dienen. Darüber hinaus erfordern die heute ver fügbaren Messtechniken teilweise umfangreiche und zeit aufwendige Datenanalysen. Schnelle, multivariate Analy senmethoden erscheinen daher unverzichtbar für eine sichere Prozesssteuerung.

Abb. 1 zeigt ein mögliches Vernetzungsschema einzelner Unit Operations zu einer verfahrenstechnischen Prozess kette und die Möglichkeit der Beeinflussung durch skalen übergreifende Simulationen sowie die Kopplung mit der Automatisierungstechnik. Ausgehend von einzelnen, verket teten Unit Operations, die nicht notwendigerweise im Batchbetrieb miteinander verbunden sein müssen, erfolgt zwischen den einzelnen Prozessschritten eine Messung der produktcharakterisierenden Eigenschaften der jeweiligen Zwischenprodukte, ausgehend von der Charakterisierung der eingesetzten Rohstoffe bis hin zum fertigen Produkt. Die gemessenen Daten dienen zur Validierung und Kalibrierung der eingesetzten Simulationen auf der partiku lären Ebene. An dieser Stelle erfolgt eine tiefgehende mathe matische Beschreibung des Prozessverhaltens mit einem sehr hohen Detailierungsgrad, wobei zur Beschreibung der Produkteigenschaften durchaus auch multiskalige Simula tionen notwendig sein können. Das Ziel von derartigen Simulationen ist die Entwicklung von Short cut Modellen, die einen wesentlich höheren Abstraktionsgrad im Vergleich zu den Simulationen auf der partikulären Ebene aufweisen, jedoch für die Berechnung der Prozesskette wesentlich schneller Vorhersagen zulassen. Ein wesentlicher Vorteil dieser Short cut Modelle besteht in der Simulation in Echt 


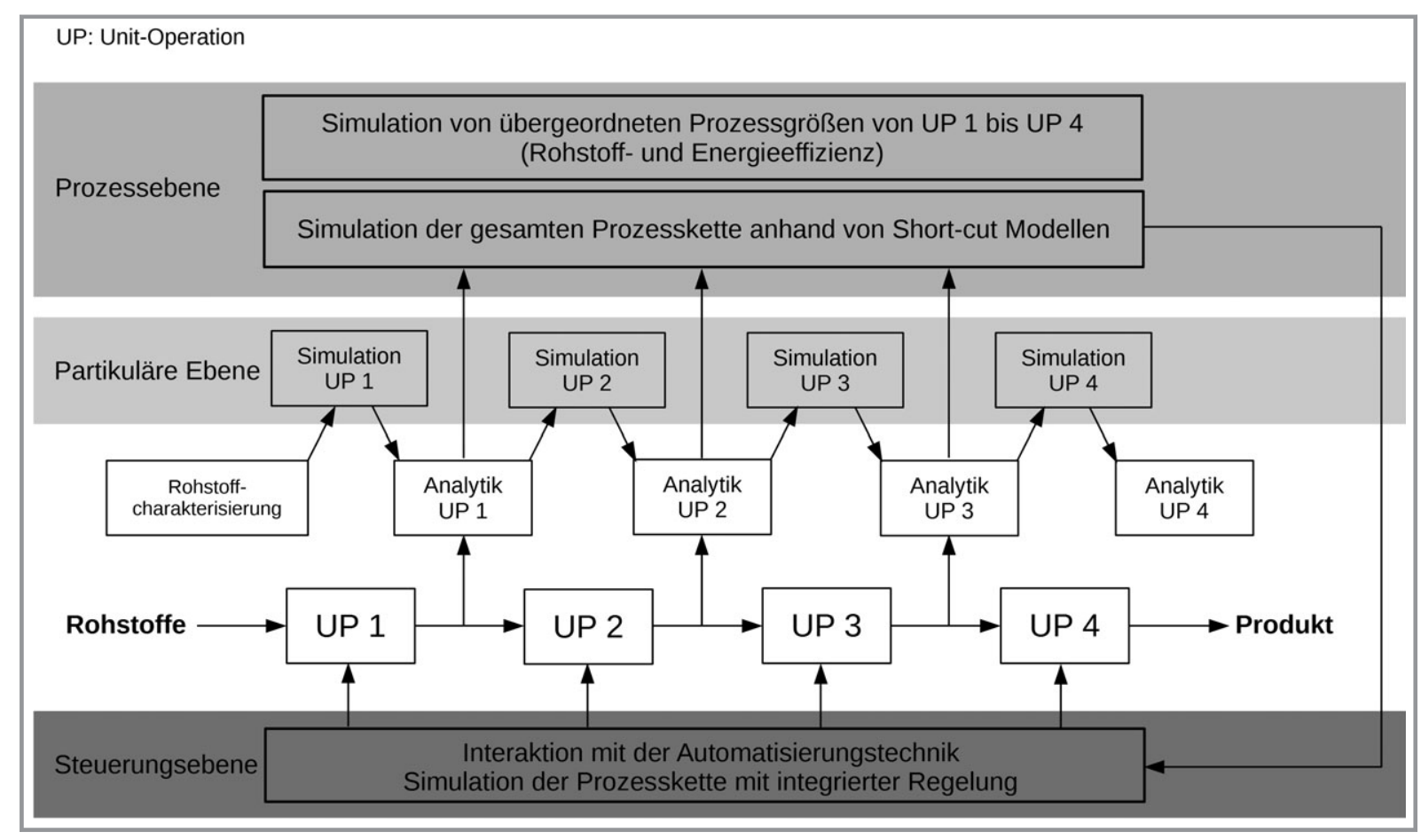

Abbildung 1. Schematische Darstellung des Vernetzungsschemas eines verfahrenstechnischen Prozesses ausgehend von Analy tik, skalenubergreifender Simulation und Verknupfung mit der Automatisierungstechnik.

zeit. Dies erlaubt die Vorausberechnung des Prozesses, um eintretende kritische Zustände durch eine frühzeitige Rege lung abzuwenden. Diese Modelle stellen auch einen Aus gangspunkt für Optimierungen dar, die eine inverse Simula tion zulassen.

Darüber hinaus ist es denkbar, auf der Prozessebene die Short cut Modelle dahingehend zu erweitern, dass überge ordnete Prozessgrößen, wie die Rohstoff und Energieeffi zienz, in die Betrachtungen mit einbezogen werden. Nicht zuletzt ist an dieser Stelle eine Kopplung zur Steuerungsebe ne denkbar. Die aus der Simulation erhaltenen Daten kön nen durch eine Datenanalyse derart aufgearbeitet werden, dass diese direkt als Stellgrößen für die Automatisierungs technik, sowohl für den realen Prozess als auch für Simulationen, dienen. Der vorliegende Artikel zeigt die beschriebene Vorgehensweise am Beispiel von partikulären Separationsprozessen für zwei Zentrifugen Typen.

\section{Grundlegende Voraussetzungen für die Simulation und digitale Kontrolle von Fest/Flüssig-Trennprozessen}

Für die Simulation sowie digitale Kontrolle und Regelung von Fest/Flüssig Trennprozessen sind neben trenntechni schen Materialeigenschaften und der Prozessanalytik zur Charakterisierung der Produkteigenschaften präzise dyna mische Prozessmodelle, auch Short cut genannt, notwendig. Abb. 2 stellt schematisch die skalenübergreifende Modellie rung für Zentrifugen als Fest/Flüssig Trennprozess dar. Die Materialfunktionen können aus Experimenten oder Simula tionen auf der Partikelebene abgeleitet werden und be schreiben die trenntechnischen Materialeigenschaften wie z. B. das Sedimentationsverhalten, die Haufwerksbildung im Zentrifugalfeld oder das rheologische Fließverhalten der gebildeten Haufwerke. Eine Herausforderung bei der Ent wicklung von Short cut Modellen ist es, mit möglichst ein fachen mathematischen Gleichungen die instationären Vor gänge bei der Separation und mechanischen Entfeuchtung präzise abzubilden. Dies setzt ein hohes Maß an Prozessver ständnis und Kenntnisse der Materialeigenschaften voraus. Im Wesentlichen beeinflussen neben den Eigenschaften der dispersen und kontinuierlichen Phase auch die Prozess bedingungen den Separationsvorgang. Zusätzlich ist neben der genauen Prozessbeschreibung auch die Recheneffizienz der dynamischen Modelle von entscheidender Bedeutung. Um während des laufenden Betriebs den Einfluss von Pro duktschwankungen und möglicher Lastwechsel vorhersagen zu können, ist es notwendig, dass die Simulationen in Echt zeit ablaufen. Mit solchen Simulationen lassen sich in einem Bruchteil von Sekunden die Zustände für einige Minuten vorhersagen, um den Prozess vorausschauend zu steuern.

Bei halb kontinuierlich und kontinuierlich arbeitenden Fest/Flüssig Trennapparaten beeinflussen die Strömungs bedingungen die Verweilzeit und dadurch den Separations vorgang. So steht jedem Partikel im Apparat eine definierte Verweilzeit für die Abscheidung zur Verfügung. Hier beein flussen sowohl der Volumenstrom und die Drehzahl als 


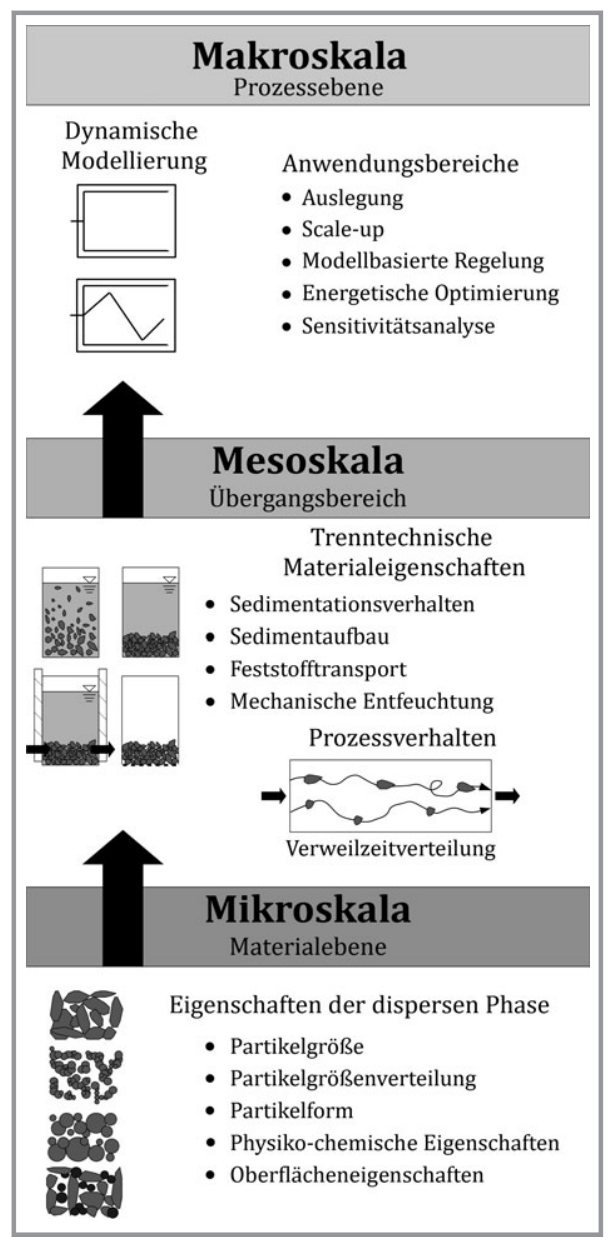

Abbildung 2. Skalenubergreifende Betrachtung der relevanten Einflussgroßen fur die Charakterisierung und mathematischen Modellierung der mechanischen Entfeuchtung von Vollmantel zentrifugen.

auch das gebildete Sediment den Strömungszustand. Eine prozessnahe Modellierung wird in diesem Zusammengang nur durch das vorhandene Prozessverständnis und die Charakterisierung der notwendigen Material und Prozess eigenschaften erreicht. Aus diesem Grund beschäftigten sich die beiden nachfolgenden Kapitel mit der notwendigen experimentellen Charakterisierung der trenntechnischen Materialeigenschaften und des Strömungszustands. Diese bilden die Grundlage für die mathematische Beschreibung der trenntechnischen Vorgänge auf der Makroskala ausge hend von einer Modellreduktion und daraus folgenden Ableitung von Short cut Modellen. An dieser Stelle sei erwähnt, dass die beschriebene Herangehensweise bei der Modellierung von Vollmantelzentrifugen auch für andere Fest/Flüssig Trennapparate wie Filterzentrifugen, Eindicker oder Filtrationsapparate denkbar ist.

\section{Notwendige Charakterisierung trenntechnischer Materialeigenschaften für numerische Simulationen}

Wie in Abschn. 2 diskutiert, ist die experimentelle Charak terisierung der Materialeigenschaften für die numerische Prozessunterstützung von entscheidender Bedeutung. $\mathrm{Zu}$ sätzlich beeinflusst die Auswahl der verwendeten trenntech nischen Eigenschaften zur Prozessbeschreibung die Güte der Simulationen und den experimentellen Aufwand.

In der Literatur werden die Eigenschaften von dispersen Systemen unter anderem in anwendungstechnische, verar beitungstechnische und physikalisch chemische Produkt eigenschaften unterschieden [9]. In dieser Arbeit wird jedoch vereinfacht nur zwischen den Eigenschaften eines Einzelpartikels und den Bulk Eigenschaften unterschieden. Die Partikelgröße, Partikelmorphologie, Dichte und die chemische Zusammensetzung zählen zu den Eigenschaften des Einzelpartikels. Durch die Anwesenheit einer Vielzahl an Partikeln in industriellen Prozessen leiten sich die fol genden prozessrelevanten Bulk Eigenschaften wie Feststoff konzentration, Sedimentationsverhalten, Kompressionsver halten des Haufwerks, Gelpunkt, Fließverhalten und Oberflächenladung ab. Dies ist nur eine Auswahl, weitere wichtige Materialeigenschaften finden sich in $[9,10]$.

Die Eigenschaften der Einzelpartikeln bestimmen sämt liche Eigenschaften in der Bulkphase. Trotzdem ist es mit dem heutigen Wissensstand nicht möglich den Einfluss von Bulk Eigenschaften auf das Prozessverhalten theoretisch aus der Charakterisierung eines Einzelpartikels abzuleiten. Der Grund hierfür liegt im Einfluss einer Vielzahl von Para metern, die wiederum untereinander wechselwirken. Zusätzlich ist die Vorhersage des Einflusses der auftretenden Partikelwechselwirkungen nicht möglich. Dadurch fehlt es allgemein an geeigneten Modellen zur Prozessunterstüt zung. Diese sind jedoch maßgeblich für die erfolgreiche Digitalisierung der mechanischen Flüssigkeitsabtrennung und anderer Bereiche der Verfahrenstechnik. Somit ist eine genaue Kenntnis der genannten Materialeigenschaften eine der Grundlagen für die Entwicklung prädiktiver Modelle zur Prozessbeschreibung. Im Folgenden werden relevante, trenntechnische Materialeigenschaften ausführlich disku tiert.

\subsection{Partikelgrößenverteilung und Feststoffkonzentration}

Die Partikelgrößenverteilung und die Feststoffkonzentra tion beeinflussen sämtliche Eigenschaften der dispersen Phasen während des Trennvorgangs [11]. Weiterhin sind das Erreichen definierter Feststoffkonzentrationen oder die Klassierung auf eine Partikelgrößenverteilungen Ziele und somit Leitparameter für eine Prozesssteuerung. Bei der Bestimmung der Partikelgrößenverteilung ergibt sich jedoch die Herausforderung, dass Partikel von der Form ei 
ner Kugel abweichen. Dadurch weisen diese Partikel keinen eindeutigen Durchmesser auf und es ist notwendig, einen Äquivalenzdurchmesser zu ermitteln. Dieser Äquivalenz durchmesser ist wiederum abhängig vom eingesetzten Messsystem $[12,13]$. Eine geeignete Wahl und Bestimmung der verwendeten Partikelgröße ist daher entscheidend für die Prozessmodellierung.

\subsection{Sedimentationsverhalten}

Das Sedimentationsverhalten beschreibt die Relativbewe gung aufgrund von Dichteunterschieden zwischen einer dispersen und einer kontinuierlichen Phase und ist somit der entscheidende Parameter bei der Modellierung des Separa tionsvorgangs. Oft wird die Relativgeschwindigkeit in Abhängigkeit der Stokes'schen Sinkgeschwindigkeit $v_{\text {Stokes }}$ eines Einzelpartikels beschrieben. Die Stokes'sche Sinkge schwindigkeit im Zentrifugalfeld ist in Gl. (1) dargelegt. Die Sinkgeschwindigkeit eines Einzelpartikels berechnet sich aus der Dichtedifferenz von Feststoff $\rho_{\text {s }}$ und der Flüssigkeit $\rho_{\mathrm{l}}$, dem Partikeldurchmesser $x$, dem Radius $r$, der Winkel geschwindigkeit $\omega$ und der Viskosität der Flüssigkeit $\eta$. Die Abweichung des Realverhaltens zur Stokes'schen Sink geschwindigkeit ist in Gl. (2) verallgemeinert dargestellt. Die Anpassungsfunktion $k$ nimmt abhängig vom gewählten Ansatz verschiedene Werte bzw. Formen an, hier sei z. B. der Ansatz von Richardson und Zaki [14] genannt.

$v_{\text {Stokes }}=\frac{\left(\rho_{\mathrm{s}} \quad \rho_{1}\right) x^{2} \omega \times(\omega \times r)}{18 \eta}$

$v_{\mathrm{korr}}=k v_{\text {Stokes }}$

\subsection{Fließverhalten}

Das Fließverhalten der flüssigkeitsgesättigten Sedimente hat einen entscheidenden Einfluss auf das Separationsergebnis. Es beschreibt die Transportierfähigkeit der abgeschiedenen Partikel im Kollektiv und somit die Sedimentverteilung im Rotor einer Vollmantelzentrifuge [15]. Abhängig der Sedi mentverteilung ergibt sich eine örtliche und zeitliche Varia tion der Strömungszustände während der Trennaufgabe. Sowohl Prozess als auch verschiedenste Materialeigen schaften beeinflussen das Fließverhalten. So ist das Fließver halten unter anderem abhängig von der Partikelgröße, der chemischen Zusammensetzung, vom Kompressionsverhal ten und der Oberflächenladung $[16,17]$. Es sind sowohl schwer als auch leichtfließende Sedimente bekannt [18]. In Abb. 3 ist schematisch der Einfluss des Fließverhaltens auf die lokale Verteilung des Sediments in einer Röhrenzentri fuge dargestellt.

Die Kenntnis des Fließverhaltens ist somit entscheidend zur Modellentwicklung bzw. Auslegung von Trennprozes

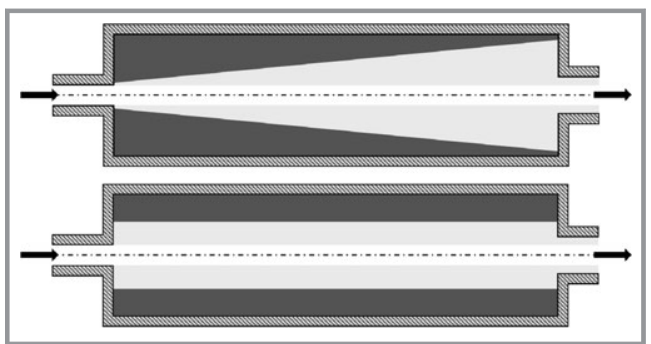

Abbildung 3. Schematische Darstellung der Sedimentvertei lung in einer Rohrenzentrifuge bei einem schwerfließenden Haufwerk (oben) und einem leichtfließenden Haufwerk (unten) nach Stahl et al. [15].

sen in Vollmantelzentrifugen. Die Charakterisierung des Fließverhaltens von flüssigkeitsgesättigten Haufwerken stellt jedoch einen interdisziplinäreren Fachbereich zwischen der Rheologie und der Schüttgutmechanik dar. In der Literatur sind Beschreibungsansätze des Fließverhaltens auf Basis von klassischen rheologischen Modellen in Kombination mit Experimenten zur Ermittlung des Fließverhaltens bekannt $[8,16]$.

\subsection{Oberflächenladung}

In Flüssigkeit dispergierte Partikel liegen infolge von Inter aktionen an der Phasengrenzfläche geladen vor. Die Ober flächenladung der dispersen Phase bestimmt die interparti kulären Wechselwirkungen, wie elektrische Anziehungs und Abstoßungskräfte. Die Ladungsstärke ist abhängig vom pH Wert der Suspension [19]. Ein Wechsel der Ober flächenladung kann zu einer signifikanten Veränderung der trenntechnischen Materialeigenschaften führen. Aus diesem Grund ist eine experimentelle Charakterisierung der in den vorherigen Abschnitten beschriebenen trenntechnischen Materialeigenschaften beim im Prozess vorliegenden $\mathrm{pH}$ Wert unabdingbar. Für eine weiterführende Beschreibung des Einflusses physiko chemischer Eigenschaften auf die trenntechnischen Materialeigenschaften sei auf Literatur von Beiser et al. [20], Nirschl [11], Erk [8], Mladenchev et al. [21] und Tiselius [22] verwiesen.

\section{Charakterisierung des Prozessverhaltens von Vollmantelzentrifugen}

Sowohl die Strömungsbedingungen innerhalb von Voll mantelzentrifugen als auch die bisher diskutierten Material eigenschaften bestimmen das Prozessverhalten. Die Strömungsbedingungen sind wiederum von den Materialei genschaften, den eingestellten Prozessparametern und der Zentrifugengeometrie abhängig. Die Herausforderung bei der Charakterisierung des Prozessverhaltens beruht auf Limitierungen in der Messtechnik und den verwendeten hohen Rotationsgeschwindigkeiten. Die Strömungsmessung 
in Vollmantelzentrifugen stellt infolge von Einbauten und der Rotationsbewegung einen komplexen Vorgang dar. In der Literatur sind einige experimentelle Strömungsunter suchungen in flüssigkeitsgefüllten Zentrifugen bekannt $[23,24]$. Jedoch ist es nicht möglich den gesamten Vorgang in der Maschine experimentell aufzulösen. Daher werden aktuell zur Beschreibung des Prozessverhaltens stark verein fachte Modelle, wie die Theorie der äquivalenten Klärfläche, genutzt [25 27]. Diese Modelle beschreiben das Grenzkorn in einer mit Flüssigkeit gefüllten Maschine unter Vernach lässigung des realen Strömungsverhaltens. Weiterhin wird der Trennapparat als Blackbox betrachtet, wodurch sich diese Ansätze ausschließlich zur Berechnung stationärer Zustände eignen. Instationäre Vorgänge wie sie bei der Durchströmung, der Sedimentation, der Haufwerksbildung oder bei Lastwechseln auftreten, finden keine Berücksichti gung. Am Institut für Mechanische Verfahrenstechnik und Mechanik (IMVM) des Karlsruher Instituts für Technologie wurden deshalb ausgehend von Analogien aus der Mess und Regelungstechnik und mithilfe der numerischen Strö mungssimulationen neue Algorithmen entwickelt, die eine prozessnahe Beschreibung transienter Vorgänge während der Separation in Vollmantelzentrifugen erlauben.

Die Grundidee ist hier das Prozessverhalten über Ände rungen der Prozessantwort zu beschreiben. Dafür wird der Prozess als Blackbox mit einer variablen Anzahl an Ein und Ausgangsströmen betrachtet [5]. Zur Untersuchung der Strömungsbedingungen eignen sich Verfahren aus der Fluidverfahrenstechnik, die zur Ermittlung des Verweilzeit verhaltens eingesetzt werden. Verweilzeitverteilungen $F$ finden eine breite Anwendung in der Verfahrenstechnik [28 31].

Die Verweilzeitverteilung ist eine indirekte Methode zur Charakterisierung der Strömungsbedingungen. Eine Mög lichkeit der Verweilzeitmessung stellt die sprunghafte Auf gabe eines Spurstoffs am Einlauf des Apparates dar. Durch die Aufzeichnung der Spurstoffänderung am Ausgang folgt als Ergebnis die Verweilzeitverteilung [32]. Aus dem Ver weilzeitverhalten lassen sich Rückschlüsse über die Strö mungsbedingungen, Totzonen und Bereiche mit hoher Rückvermischung ziehen. Je breiter eine Verweilzeitvertei lung, umso größer ist der Einfluss axialer Dispersion. Eine ausführliche Diskussion der Vor und Nachteile dieser Betrachtungsweise geben Platzer et al [33].

In Abb. 4 sind beispielhaft die auf die mittlere Verweilzeit normierten Verweilzeitverteilungen eines idealen konti nuierlichen Rührreaktors (CSTR), eines idealen Pfropfenst romreaktors (PFR) und eines nicht idealen Prozesses darge stellt [32]. Ein idealer CSTR stellt ein System mit maximaler Rückvermischung dar. In einem idealen PFR tritt keine Rückvermischung auf. Das reale Verhalten liegt in den meisten Fällen zwischen den beiden beschriebenen Grenz fällen. Zusätzlich ist die Kenntnis der zeitlichen Reaktion des Gesamtsystems auf Änderungen von Prozessparame tern und Materialeigenschaften, wie die Partikelgrößenver teilung oder Feststoffkonzentration, entscheidend für die

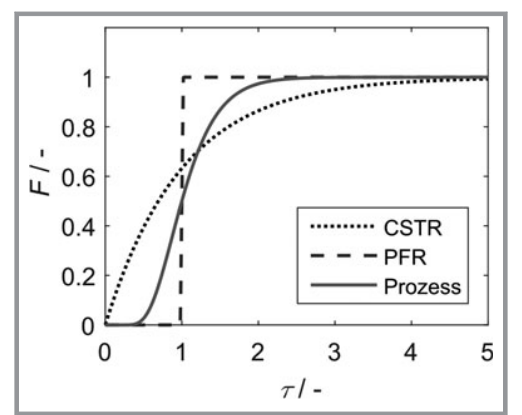

Abbildung 4. Vergleich der Verweilzeitverteilungen $F$ eines idealen kontinuierlichen Ruhrkessels, eines idealen PFR und dem realen Verweilzeitverhalten bei der Separation. Die Durch stromungsziffer $\tau$ entspricht der auf die mittlere Verweilzeit bezogenen Zeit.

Prozessmodellierung. Die Bestimmung erfolgt dabei analog zu Verweilzeitverteilungen ausgehend von der sprunghaften Änderung von Systemgrößen.

\subsection{Aufgelöste CFD-Simulationen zur Untersuchung des Prozessverhaltens}

Die experimentellen Untersuchungen sind nur ein Schritt auf dem Weg zum tieferen Prozessverständnis. Sie liefern keine detaillierten Informationen über den zeitlichen Ver lauf des Füllgrads oder die Sedimentverteilung entlang des Rotors. Durch die wachsende Rechenleistung halten CFD Simulationen Einzug in einige Bereiche der Verfah renstechnik. In der Literatur sind inzwischen sowohl eigen entwickelte als auch Standardmethoden zur numerischen Beschreibung von Mehrphasenströmungen und zur Strö mungsuntersuchung in Zentrifugen bekannt [34 38]. Um auch in der mechanischen Flüssigkeitsabtrennung für semi batch oder kontinuierlich betriebene Vollmantelzentrifugen aufgelöste Simulationen als Unterstützung zu experimentel len Untersuchungen nutzen $\mathrm{zu}$ können, befasst sich das IMVM seit einiger Zeit mit der räumlichen und zeitlichen Auflösung des Strömungsfelds. Dabei steht die Entwicklung von neuen Ansätzen basierend auf der numerischen Strömungssimulation (CFD) im Vordergrund. Hier werden die Navier Stokes Gleichungen mit dem Fließverhalten flüssigkeitsgesättigter Haufwerke verknüpft und so der Trennprozess aufgelöst dargestellt [39].

Allgemein ist die Handhabung einer großen Anzahl an Partikeln die Herausforderung bei Simulationen in der Par tikeltechnik. Bei Vollmantelzentrifugen treten außerdem hohe Strömungsgeschwindigkeiten auf. Zusätzlich ändert sich das Materialverhalten am Übergang von dispergierten Partikeln zu einem Haufwerk. Weiterhin kommt es zu einer Wechselwirkung zwischen Maschinen und Materialver halten. Gleichzeitig erfordern die hohen Strömungs geschwindigkeiten und die Vielzahl an physikalischen Vorgängen eine möglichst in Bezug auf die Rechenzeit effi ziente Vorgehensweise. Allein die hohen Strömungs 
geschwindigkeiten bedingen einen Zeitschritt im Bereich von $\Delta t \approx 10^{-4} \quad 10^{-6} \mathrm{~s}$ für stabile numerische Simulationen. Somit ist ein Kompromiss zwischen physikalischer Auf lösung der trenntechnischen Vorgänge und des Rechenzeit bedarfs notwendig.

Eine weitverbreitete numerische Methode zur Beschrei bung von Mehrphasenströmungen ist der Euler Euler Ansatz. Hier werden die einzelnen Phasen als wechsel wirkende Kontinua betrachtet. Die Wechselwirkungen zwischen den Phasen sind mittels Quellen und Senken beschrieben [34]. Die Berücksichtigung der Partikelgrößen verteilung erfordert das Lösen der Massen und Impulser haltung für jede Größenklasse, was aktuell einen nicht zu lösenden Rechenaufwand darstellt. Die hohe Anzahl an Par tikeln im Rotor lässt außerdem keine Anwendung der Euler Lagrange Methode zu [34]. Somit sind klassische Ansätze zu rechenintensiv, um den Trennprozess innerhalb von Vollmantelzentrifugen mit ausreichender Genauigkeit und die vorhandenen Zeitskalen abzubilden.

Das Zwei Phasen System bestehend aus disperser Phase und Flüssigkeit wird mit dem am IMVM entwickelten Modell als Mischphase modelliert [39]. Dieser Ansatz ist dem sogenannten Fast Eulerian Approach von Ferry et al. [40] angepasst. Mit der Implementierung einer Partikelklas se erweitert sich dieses Modell jeweils um eine Transport gleichung für den Feststoffvolumenanteil. Die Beschreibung der Rückwirkung dispersen Phase auf die Strömung erfolgt nicht über Quellterme, sondern mittels ortsabhängigen Viskositätsfunktionen, die zwischen Suspension und Sedi ment unterscheiden. Die Modellierung der instationären Strömungsbedingungen durch einen quasi stationären Ansatz verringert zusätzlich den benötigten Rechenauf wand. Eine detailliere Beschreibung der Simulationsmetho de zeigen Hammerich et al. [39]. Weiterhin ist das Kom pressionsverhalten flüssigkeitsgesättigter Haufwerke im mathematischen Modell eingebunden und erlaubt so die Simulation des Realbetriebs.

Trotz sämtlicher Maßnahmen zur Reduktion der Rechen dauer ergibt sich je nach Rotationsgeschwindigkeit, Partikel anzahl und der Größe des Rechengitters eine Simulations zeit im Bereich von einigen Stunden bis hin zu mehreren Tagen. Grund dafür ist die bereits diskutierte kleine Zeit schrittweite infolge der hohen Geschwindigkeitsgradienten. Die entwickelte Simulationsmethode stellt einen Kompro miss zwischen physikalischer Auflösung der trenntechni schen Vorgänge und des Rechenzeitbedarfs dar. Dies erlaubt es, die gesamte Maschine in Kombination mit den trenn technischen Materialeigenschaften zu simulieren. Ein Ein satzgebiet dieser Methode ist die Optimierung der Zentrifu gen Geometrie und die Ableitung von Short cut Modellen zur Beschreibung der Prozessebene. Im Falle dieser Arbeit ist der Einfluss des Sediments auf die Strömungsbedingun gen und somit auf das Verweilzeitverhalten von Interesse.

In Abb. 5 sind beispielhaft vier ermittelte Verweilzeitver teilungen mit den dazugehörigen Sedimentprofilen und Strömungszuständen abgebildet. Dabei strömt die Suspen sion von der linken Seite nach rechts in Richtung des Über laufs. Der Einfluss des sich bildenden Sediments auf die Strömung und somit auf die Verweilzeitverteilungen ist gut zu erkennen. Zum Startzeitpunkt entspricht der Zustand einer flüssigkeitsgefüllten Röhrenzentifuge. Mit der Zeit baut sich das Sediment an der Rotorinnenwand auf. Wie zu erwarten, verschieben sich die Verweilzeitverteilungen mit steigendem Füllgrad zu kleineren Verweilzeiten.

Gleichzeitig lassen sich Informationen über Einflussfako ren und Prozesszustände ableiten, die experimentell meist nicht zugänglich sind. Einen solchen Parameter stellt der Einfluss des Fließverhaltens auf die Sedimentverteilung dar.

Auf der linken Seite in Abb. 6 ist der Einfluss des Fließ verhaltens auf die Strömung innerhalb des Rotors für einen Zeitschritt von $t=100 \mathrm{~s}$ dargestellt. Im gezeigten Fall strömt die Suspension von links nach rechts. Das Fließverhalten für den Zustand 1 (Z1) entspricht einem schwerfließenden Haufwerk. Zustand 2 (Z2) stellt das Fließverhalten eines leichtfließenden Haufwerks dar. An dieser Stelle sei ange merkt, dass in Abb. 6 sowohl die Geometrie als auch die Prozessbedingungen konstant sind. Die Unterschiede in der Sedimentverteilung und im Strömungszustand sind hier auf

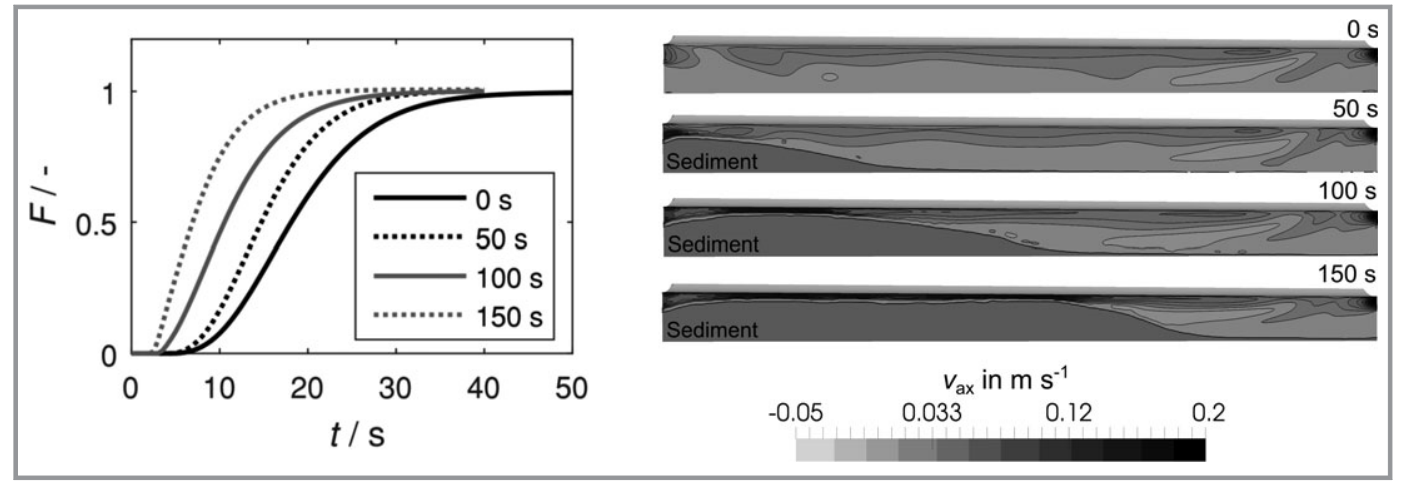

Abbildung 5. Links: Verweilzeitverteilung $F$ in Abhangigkeit der Zeit fur vier Zeitschritte $t=0 \mathrm{~s}, 50 \mathrm{~s}, 100 \mathrm{~s}$ und 150 s der numerischen Simulation. Rechts: Darstellung der Sedimentkontur und der Veranderung der axialen Komponente der Stromungsgeschwindigkeit $v_{\mathrm{ax}}$ fur die bereits beschriebenen Zeitschritte. 
das eingestellte Fließverhalten zurückzuführen. Das Dia gramm auf der rechten Seite in Abb. 6 zeigt die Verweilzeit verteilung in Abhängigkeit der Durchströmungsziffer $\tau$, die das Verhältnis aus aktuellem Zeitschritt zur mittleren Ver weilzeit beschreibt. Beim Vergleich der beiden Verteilungen untereinander sowie mit der Ausgangsverteilung fällt auf, dass abhängig vom Sedimentaufbau in der Zentrifuge sich die Breite der Kurven ändert. Bei einem schwerfließenden Sediment liegt eine inhomogene Sedimentverteilung vor und es kommt infolge der schnellen Aufweitung des Quer schnitts am Übergang zu einer stärkeren Rückvermischung und somit axialen Dispersion. Beim leichter fließenden Sediment ist das Sediment gleichmäßig an der Rotorinnen wand verteilt und hat damit eine geringe axiale Rückvermi schung zur Folge.

\section{Dynamische Modellierung der Prozessebene und Echtzeit-Simulationen}

In der Literatur sind eine Reihe von Ansätzen zur Beschrei bung der Separation und der Kuchenbildung im Erdschwe re sowie Zentrifugalfeld vorhanden. Die Grundlage der meisten Modelle bildet das Kynch Theorem [41]. Kynch trifft die Annahme, dass das Sedimentationsverhalten von der lokalen Feststoffkonzentration abhängt. Ausgehend von einer differentiellen Bilanz um ein Volumenelement ergibt sich daraus der zeitliche Verlauf der Feststoffkonzentration entlang der wirkenden Feldkraft. Garrido et al. [42], Bürger et al. [43] und Stickland et al. [44] erweitern die Kynch Theorie auf den Kuchenbildungsvorgang eines kompressib len Haufwerks sowohl für das Erdschwere als auch das Zentrifugalfeld. Das resultierende Gleichungssystem hat die Form einer hyperbolisch parabolischen Differentialglei chung, die ausschließlich mittels numerischer Methoden lösbar ist. Die Schwierigkeit in der Beschreibung des Sedi mentationsvorgangs liegt im sprunghaften Übergang des
Materialverhaltens von der Suspension zum Haufwerk. Dies führt zu kleinen Zeitschrittweiten, was keine Echtzeit Simu lationen der trenntechnischen Vorgänge erlaubt. Es sei an dieser Stelle angemerkt, dass Bürger et al. [43] und Stick land et al. [44] ausschließlich die Batch Zentrifugation im Labor Maßstab betrachten. Eine Übertragung auf Prozess zentrifugen oder den industriellen Maßstab wurde nicht durchgeführt.

Echtzeit Simulationen sind eine weitere Voraussetzung für die Anwendung dynamischer Prozessmodelle auch zur modellbasierten Regelung in der mechanischen Flüssigkeits abtrennung. Beispiele für derartige dynamische Modelle sind bisher nur für statische Eindicker bekannt. Takacs et al. [45] und Zheng et al. [46] stellen die Aufkonzentrie rung einer Suspension im statischen Eindicker mittels eines Ersatzschaltbildes dar. Für die mathematische Modellierung wird der Trennapparat in eine definierte Anzahl an Schich ten entlang der wirkenden Feldkraft unterteilt und für jede Schicht eine Bilanzgleichung sowohl für den Feststoff als auch für die Flüssigkeit aufgestellt. Für die prozessnahe Beschreibung von statischen Eindickern wird das Sedimen tationsverhalten in Abhängigkeit der Feststoffkonzentration im Labormaßstab gemessen und daraus Short cut Modelle für die Sinkgeschwindigkeit abgeleitet [47].

Der Detailgrad der Modellierung von Prozesszentrifugen steigert sich im Vergleich zu statischen Eindickern und der Batch Zentrifugation deutlich. Der Grund ist im abweich enden Strömungsverhalten der betriebenen Apparate zu finden [48]. Die Hauptströmungsrichtung des Fluids ist nicht wie für Eindicker entgegengesetzt der Sedimentation, sondern in Richtung des vorhandenen Überlaufwehrs. Durch die wirkende Zentrifugalkraft wandern die Teilchen in radialer Richtung und werden gleichzeitig durch die Fluidströmung in Richtung Überlauf transportiert. Infolge dessen kommt es zu einer Querstromklassierung entlang des Rotors [49]. Das am IMVM entwickelte dynamische Prozessmodell gibt die Möglichkeit, Vorhersagen über die

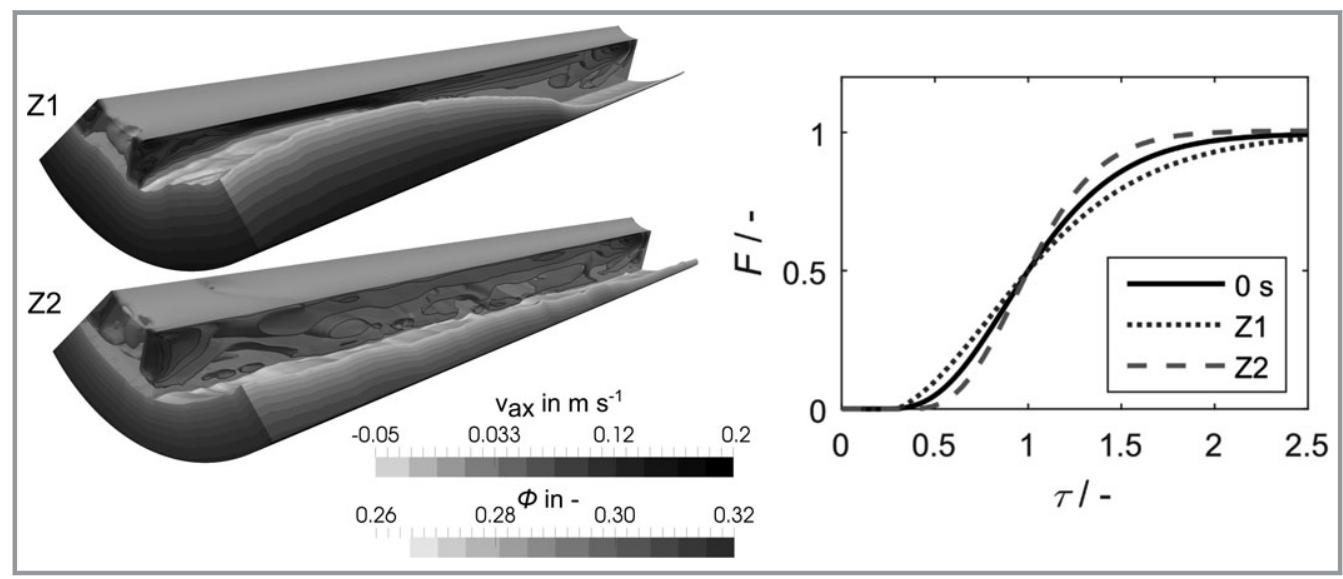

Abbildung 6. Links: Axiale Stromungsgeschwindigkeit $v_{\mathrm{ax}}$ und Sedimentverteilung entlang des Rotors unter Variation des Fließverhaltens. Fließverhalten Z1 entspricht einem schwerfließenden Sediment, Fließverhalten Z2 einem leichtfließenden. Rechts: Verweilzeitverteilung $F$ der unterschiedlichen Zustande als Funktion der Durchstromungsziffer $\tau$. 
Trennleistung und das Entfeuchtungsverhalten unterschied licher Zentrifugen Typen und Baugrößen zu treffen. Ein Ersatzschaltbild kombiniert hier die Maschinenfunktion und die trenntechnischen Materialeigenschaften. Es sei angemerkt, dass die Maschinenfunktion das Verweilzeitver halten beschreibt und sich aus Experimenten oder CFD Simulationen ergibt. Die trenntechnischen Materialeigen schaften bilden das Sedimentationsverhalten, den Kuchen bildungsprozess und den Sedimenttransport ab.

Abb. 7 zeigt schematisch den Aufbau der dynamischen Prozessmodelle für Dekantier und Röhrenzentrifugen. Bei de Prozesszentrifugen unterscheiden sich in erster Linie durch den Aufbau und das Prozessverhalten. In Dekantier zentrifugen bewegt sich der vorhandene Schneckengrund körper mit einer Relativgeschwindigkeit zur Zentrifugen trommel und fördert das gebildete Haufwerk kontinuierlich aus dem Verfahrensraum [50]. In Röhrenzentrifugen hinge gen ist keine Förderschnecke vorhanden und nach Errei chen des maximalen Füllgrads wird der Apparat herunter gefahren und entleert. Betrachtet man den Stand der Technik, stellt man fest, dass eine Reihe von Einflussgrößen, wie der Impulsaustausch zwischen Feststoff und Flüssigkeit, der Transport der flüssigen und dispersen Phase, die Hauf werksbildung und den Sedimenttransport, nicht berück sichtigt werden $[25,26]$. Dadurch ist ein Scale up nur in einem definierten Größenbereich und Parameterfeld unter

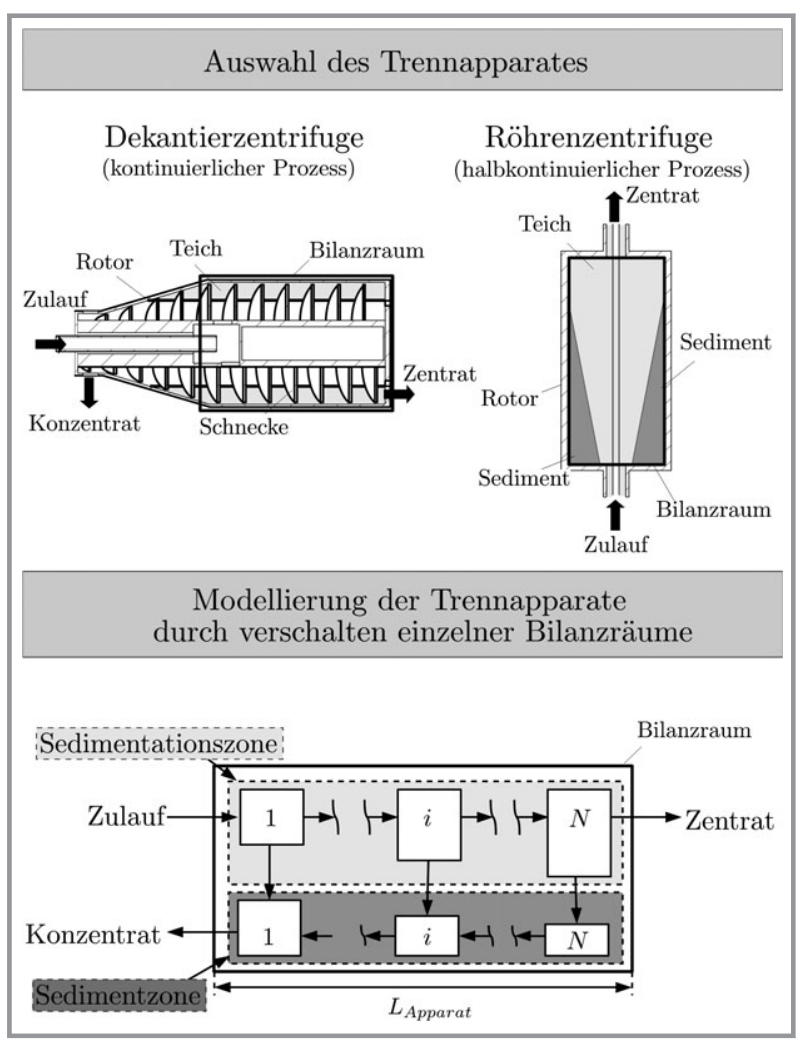

Abbildung 7. Schematische Darstellung des Bilanzraum An satzes fur Rohrenzentrifugen bestehend aus der Sedimenta tions und Sedimentzone.
Berücksichtigung von einer Vielzahl von Versuchen im Pilot Maßstab möglich [27]. Eine weitere Schwierigkeit ist die Kopplung zwischen Strömungsbedingungen und trenn technischem Materialverhalten. Hier führt die Haufwerks bildung zu einer Reduzierung des freien Strömungsquer schnitts und damit zu einer Verringerung der Verweilzeit im Trennapparat.

Der untere Teil in Abb. 7 stellt den von Gleiss et al. [5] beschriebenen Bilanzraum Ansatz für die Schneckenab wicklung des zylindrischen Teils einer Gegenstrom Dekan tierzentrifuge dar. Für die Berechnung wird der Strömungs kanal entlang der Schneckenhelix in eine Sedimentations und die Sedimentzone unterteilt und das Separationsverhal ten, die Haufwerksbildung und der Sedimenttransport in Echtzeit berechnet [5]. Die Grundlage der mathematischen Modellierung bildet das Lösen von Massen und Kompo nentenbilanzen für den Feststoff und die Flüssigkeit in Kombination mit Short cut Modellen für das Separations verhalten und die Kuchenbildung. Weiterhin wird die Men genbilanz für jede Partikelgrößenklasse in den einzelnen Bilanzräumen gelöst.

Wie Beiser [51] und Gleiss et al. [5,52] beschreiben, ist die Trenngradfunktion ein Short cut Modell zur mathema tischen Beschreibung der Klassierung in Dekantierzentrifu gen. Beiser [51] geht in seinem Modell von einer axialen Kurzschlussströmung entlang der zylindrischen Trommel aus. Im Realbetrieb findet die Hauptströmungsrichtung in Dekantierzentrifugen entlang des Schneckenkanals statt. Gleiss et al. [5,52] leiten aus dieser Beobachtung den Trenngrad entlang der Schneckenabwicklung her. Der Trenngrad hängt dabei von der Zentrifugen Geometrie, den Prozessbedingungen und den Materialeigenschaften ab. Die Formulierung des Gleichungssystems liefert eine gewöhn liche Differentialgleichung 1. Ordnung, welche die zeitliche Änderung der Feststoffkonzentration im Apparat beschreibt [52]. Die Lösung des beschriebenen Gleichungssystems erfolgt numerisch mittels eines fünfstufigen Runge Kutta Verfahren in der Softwareumgebung Matlab.

Im Gegensatz zum Stand der Technik wird außerdem der Sedimentaufbau und der Sedimenttransport bei der Berech nung von Dekantierzentrifugen berücksichtigt [4]. Das Lösen der Massenbilanz der Feststoffphase ergibt die Mas senverteilung des Sediments entlang des Schneckenkanals. Das vorhandene Sediment führt zu einer Reduzierung des freien Strömungsquerschnitts. Im Gegensatz zum Stand der Technik erfolgt durch die Diskretisierung des Verfahrens raums die Berechnung der Feststoffkonzentration und der Partikelgrößenverteilung als lokal verteilte Größe. Für den Fall der dynamischen Simulation einer Röhrenzentrifuge werden die gleichen mathematischen Modelle unter Ver nachlässigung des Sedimenttransports verwendet. 


\subsection{Dynamische Simulationen auf der Makroskala am Beispiel zweier Zentrifugen-Typen}

Abb. 8 zeigt den Vergleich von Experiment und dynami scher Simulation für den zeitlichen Verlauf des Abscheide grads einer Röhrenzentrifuge im Technikumsmaßstab mit einem Gesamtvolumen von $V=250 \mathrm{~mL}$. Im dargestellten Fall gilt für den Volumenstrom $Q_{2}>Q_{1}$. Während des Anfahrvorgangs füllt sich die Maschine bis das Überlauf wehr erreicht wurde. Anschließend erfolgt der Austrag eines Teils der dispersen Phase über das Überlaufwehr und es kommt zu einem Abfall des Abscheidegrads. Im weiteren Verlauf sinkt die Verweilzeit durch den kontinuierlichen Sedimentaufbau in der Röhrenzentrifuge. Gegen Ende des betrachteten Zeitraums ist der Apparat fast vollständig mit Sediment gefüllt und der Abscheidegrad sinkt auf $A=0$. Dies ist führt dazu, dass der zugeführte Feststoff die Röh renzentrifuge vollständig verlässt und infolge der geringen Verweilzeit kein weiterer Feststoff akkumuliert. Beim freien Strömungsquerschnitt in der Röhrenzentrifuge handelt es sich um eine dünne Kreisringfläche nahe des Flüssigkeits teichs in der hohe axiale Strömungsgeschwindigkeiten auf treten. Weiterhin zeigt sich, dass eine Erhöhung des Volu menstroms eine Veränderung der Gesamtprozesszeit und der Abscheideleistung herbeiführt. Abschließend lässt sich fest stellen, dass das dynamische Modell den zeitlichen Verlauf des Abscheidegrads mit einer guten Genauigkeit abbildet.

Das dargestellte nicht lineare Prozessverhalten führt dazu, dass konventionelle PI Regler durch die Veränderung der Verweilzeit über die Gesamtprozessdauer an ihre Gren zen stoßen $[53,54]$. In den meisten Fällen wird für diese Modelle aus der Sprungantwort eine Zeitkonstante abgelei tet. Diese Zeitkonstante ist jedoch für Röhrenzentrifugen nicht konstant, sondern eine Funktion der Zeit. Durch die zeitliche Änderung der Ausgangsgröße und des gezeigten nicht linearen Verhaltens ist eine Abschätzung der Zeitkon stante während des Betriebs nicht durchführbar. Eine Alter native zu konventionellen PI oder PID Reglern stellt die modellbasierte Regelung dar. Durch die Echtzeit Simulation mittels des mathematischen Modells ist es denkbar, den ge zeigten numerischen Algorithmus in einen An satz zur modelbasierten Regelung einzubinden. Hierzu müssen die Feststoffkonzentration und die Partikelgrößenverteilung als Eingangs und Ausgangsgrößen mittels geeigneter Prozessanaly tik gemessen werden. Das dynamische Modell er laubt es anschließend in Kombination mit den Messwerten den Separationsvorgang in der Röh renzentrifuge vorauszuberechnen und anschlie ßend die Verweilzeit zum jeweiligen Zeitpunkt abzuleiten. Das Setzen eines Kriteriums für die Änderung des Ausgangssignals gestattet die An passung der Drehzahl oder des Volumenstroms über die Gesamtprozesszeit.

Die linke Seite in Abb. 9 zeigt den zeitlichen Verlauf der Feststoffkonzentration $\Phi$ am Zulauf

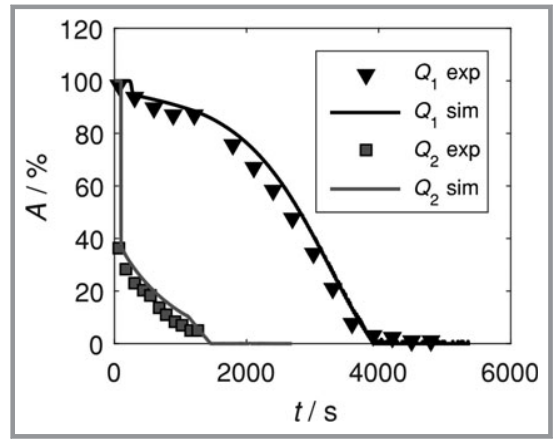

Abbildung 8. Zeitliche Verlaufe des Abscheidegrads $A$ wahrend der Separation von Aerosil 200 in einer Rohrenzentrifuge fur unterschiedliche Volumenstrome $Q$. Vergleich der Echtzeit Simulationen mit experimentellen Daten von Spelter [55].

einer Dekantierzentrifuge im Labormaßstab. Die Feststoff konzentration stellt im betrachteten Fall einen Eingabepara meter der dynamischen Simulation dar und ist als zeitab hängige Größe am Zulauf definiert. Im für die numerische Studie vorgegebenen Fall kommt es nach einer Zeit von $t=$ $500 \mathrm{~s} z u$ einem linearen Anstieg der Feststoffkonzentration, bis sich der Verlauf nach einer Zeit von $t=800 \mathrm{~s}$ nicht mehr ändert. Schwankungen der Feststoffkonzentration am $\mathrm{Zu}$ lauf können im Realbetrieb durch Produktschwankungen im Partikelbildungsprozess entstehen.

Auf der rechten Seite in Abb. 9 ist der zeitliche Verlauf des Abscheidegrads $A$ sowie des Zulaufvolumenstroms $Q$ für den nicht geregelten und den geregelten Fall dargestellt. Dabei findet bei den eingestellten Anfangsbedingungen eine vollständige Klärung der Suspension statt und der Abschei degrad beträgt $A=100 \%$. Infolge der Änderung der Fest stoffkonzentration am Zulauf ergibt sich eine Änderung der trenntechnischen Materialeigenschaften in der Dekantier zentrifuge. Konkret bedeutet dies, dass der Einfluss hydro dynamischer Wechselwirkungen zwischen disperser Phase und Flüssigkeit ansteigt. Also Folge kommt es zu einer Ver ringerung der Sinkgeschwindigkeit und bei konstanten Pro zessbedingungen strömt mehr Feststoffmasse am Zulauf in die Zentrifuge. Als Ergebnis steigt der volumetrische Füll

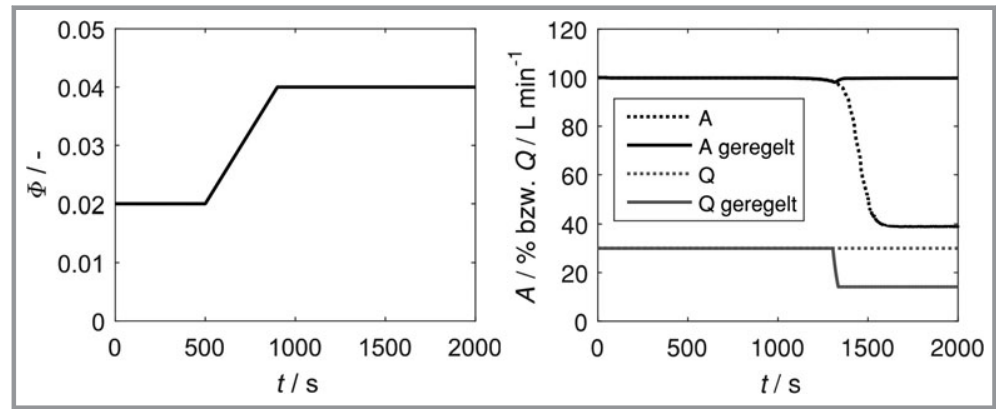

Abbildung 9. Numerische Studie zum zeitlichen Verlauf des Abscheidegrads $A$ in einer Gegenstrom Dekantierzentrifuge. Links: Eingangssignal der Feststoff konzentration $\Phi$. Rechts: Abscheidegrad $A$ und nicht geregelter und geregelter Volumenstrom $Q$ als Funktion der Zeit $t$ nach linearer Erhohung der Feststoff konzentration [52]. 
grad über die Zeit an. Durch die Änderung der trenntechni schen Eigenschaften tritt nicht sofort eine Reaktion der Ma schine auf diesen Lastwechsel ein. Im nicht geregelten Fall ist die Reaktion erst nach einer Zeit von $t=1300 \mathrm{~s}$ sichtbar. Als Grund kann hier die Verschiebung des Verhältnisses aus Sedimentationszeit und Verweilzeit in Richtung kleinerer Verweilzeiten durch den Sedimentaufbau genannt werden. Als Folge kommt es bei konstanten Prozessbedingungen zu einem erhöhten Produktaustrag, was im Falle der vollstän digen Klärung der Maschine nicht erwünscht ist.

Das dynamische Modell beschreibt die Prozessbedingun gen und die Materialeigenschaften am Zulauf als zeitabhän gige Größen. So wurde für den geregelten Fall das Ausgang signal mit der Zeit aufgezeichnet und nach Erreichen eines kritischen Wertes der Volumenstrom so angepasst, dass kei ne Verschlechterung während der Klärung der Suspension eintritt. Für den geregelten Fall verringert das dynamische Modell daher schrittweise den Volumenstrom, was einen Anstieg der Verweilzeit der Suspension in der betrachteten Dekantierzentrifuge zur Folge hat. Als Ergebnis bleibt der Abscheidegrad näherungsweise konstant und ein effizienter Betrieb bei vollständiger Klärung ist gewährleistet.

Zusammenfassend zeigt sich bei der Betrachtung des Separationsvorgangs für beide beschriebenen Zentrifugen Typen, dass ein deutlich voneinander abweichendes Pro zessverhalten vorhanden ist. Konventionelle stationäre Modelle, die den Stand der Technik darstellen, erlauben keine zeitabhängige Berechnung des Separationsvorgangs. Dies ist jedoch für die Vorhersage von Lastwechseln zwin gend erforderlich. Ein weiterer Vorteil des Ansatzes ist die orts und zeitaufgelöste Betrachtung des Separationsvor gangs für die beschriebenen Zentrifugen Typen und die Durchführung von Sensitivitätsanalysen. Dadurch wird es möglich, den Einfluss unterschiedlicher Parameter am Rechner vorherzusagen, um so einerseits ein besseres Pro zessverständnis aufzubauen und andererseits dieses Wis sen für die gezielte Schulung neuer Mitarbeiter einzuset zen. Durch die Verknüpfung dieser Ansätze mit künstlicher Intelligenz ist es weiterhin denkbar, selbstler nende Regler aufzubauen, die datenbasiert gemessene Größen aus dem Prozess ableiten und damit die modell basierte Regelung gezielt unterstützen.

\section{Zusammenfassung und Ausblick}

Der vorliegende Beitrag zeigt die skalenübergreifende mathematische Modellierung der Zentrifugation als Trenn schritt in der Fest/Flüssig Trennung exemplarisch am Bei spiel von Röhren und Dekantierzentrifugen. Beim Über blick über mögliche Einflussgrößen ergibt sich ein vielfältiges Parameterfeld, das von den Eigenschaften der dispersen und kontinuierlichen Phase abhängt. Mögliche Einflussparameter sind die Partikelgröße, die Partikelform oder die physiko chemischen Eigenschaften.
Die mathematische Modellierung der Strömungsvorgänge mittels aufgelöster CFD Simulation in Kombination mit dem Fließverhalten der Suspension und des flüssigkeitsge sättigten Haufwerks erlaubt die Beschreibung der komple xen Wechselwirkungen zwischen Flüssigkeit und Feststoff. Zur Berücksichtigung des Strömungsverhaltens auf der Pro zessebene wurde eine Modellreduktion durchgeführt und ein Short cut Modell zur Beschreibung des Verweilzeitver haltens abgeleitet. Zur Echtzeit Simulation der mechani schen Flüssigkeitsabtrennung in Prozesszentrifugen wird das Verweilzeitverhalten mittels eines Ersatzschaltbildes modelliert und mit den trenntechnischen Materialeigen schaften der Sedimentation, der Kuchenbildung und des Feststofftransports verknüpft. Dabei zeigt sich für Dekan tier und Röhrenzentrifugen ein abweichendes Prozessver halten. Während sich für Dekantierzentrifugen nach der Anfahrphase ein stationärer Zustand einstellt, kommt es für Röhrenzentrifugen zu einer kontinuierlichen Änderung der Strömungsbedingungen und der trenntechnischen Mate rialeigenschaften.

Für zukünftige Arbeiten ergeben sich eine Reihe von Fra gestellungen. So fehlt es an Online oder In situ Messver fahren, um nanoskalige Partikelsysteme während des Sepa rationsvorgangs mit ausreichender Genauigkeit messen zu können. Dies ist jedoch eine wichtige Voraussetzung bei der Entwicklung von Algorithmen zur modellbasierten Rege lung und numerischen Optimierung des Trennschritts der Zentrifugation. Weiterhin fehlt es an Short cut Modellen, welche die Sedimentation, den Sedimentaufbau und das Fließverhalten in Abhängigkeit der Partikelgrößenvertei lung und physiko chemischen Eigenschaften darstellen. Dies ist jedoch eine wichtige Voraussetzung, um den experi mentellen Aufwand bei der Analyse im Labor und Pilot maßstab gering zu halten. Außerdem fehlt es an numeri schen Algorithmen, die eine Regelung des Prozesses ausgehend von Online und In situ Messungen in Kombi nation mit Echtzeit Simulationen erlauben. Die Kombina tion der einzelnen, beschriebenen Punkt würde es in einem weiteren Schritt erlauben einen digital twin (digitales Abbild) für den Trennschritt der Zentrifugation zu Erstellen und so einen automatischen und sicheren Betrieb in der Prozessindustrie zu gewährleisten.

Die Autoren bedanken sich bei der Deutschen Forschungsgemeinschaft für die finanzielle Unterstüt zung im Rahmen des Schwerpunktprogramm SPP1679 „Dynamischen Simulation vernetzter Feststoffprozesse DynSim FP“ (NI 414/21). Weiterhin bedanken sich die Autoren bei der Allianz Industrie und Forschung für die finanzielle Unterstützung in den IGF Vorhaben 18461N und $19528 \mathrm{~N}$. 
Simon Hammerich absol vierte bis 2013 den Diplom studiengang Bioingenieur wesen am Karlsruher Insti tut für Technologie (KIT). Seit Ende 2013 ist er wis senschaftlicher Mitarbeiter am Institut für Mechani sche Verfahrenstechnik und Mechanik am KIT in der Arbeitsgruppe von Prof. Dr. Ing. habil. Hermann Nirschl. Im Zuge seiner Promotion beschäftigt er sich seit 2014 mit der numerischen Simulation von Separationsprozessen in Vollmantelzentrifugen und der Charakterisierung des Fließverhaltens gesättigter Haufwerke.

Marco Gleiß studierte Ver fahrenstechnik am Karlsru her Institut für Technologie (KIT) und promovierte 2018 am Lehrstuhl für Mechanische Verfahrens technik und Mechanik des KIT zum Thema der Dyna mischen Modellierung von Vollmantelzentrifugen. Seit 2018 arbeitet er als Post Doc in der Arbeitsgruppe von Prof. Dr. Ing. habil.

Hermann Nirschl und beschäftigt sich mit der numeri schen Simulation, der modellbasierten Regelung, der dynamischen Fließschemasimulation sowie der Entwicklung von Analysemethoden zur Messung von Partikeleigenschaften im Bereich der mechanischen Flüssigkeitsabtrennung.
Hermann Nirschl promo vierte $1994 \mathrm{im}$ Bereich der Fluiddynamik an der Tech nischen Universität Mün chen. Bis 1997 habilitierte er im Bereich der numeri schen Simulation von parti kelbeladenen Strömungen. Von 1997 bis 2002 war er Leiter der Verfahrenstech nik im Bereich der Dental sparte der Firma 3M und fungierte als Projektmana ger für verschiedenste Projekte in München und St. Paul/Minnesota. Seit 2003 ist er Professor für Mecha nische Verfahrenstechnik am Karlsruher Institut für Technologie. Der Fokus seiner Forschung liegt im Bereich der Partikeltechnik mit dem Schwerpunkt auf Separationsprozesse, numerischen Simulationen und der Entwicklung von Methoden und Geräten zur Partikelanalyse.

\section{Formelzeichen}

$\begin{array}{lll}A & {[\mathrm{]}} & \text { Abscheidegrad } \\ F & {[\mathrm{]}} & \text { Verweilzeitverteilung } \\ k & {[\mathrm{]}} & \text { Anpassungsfunktion } \\ m & {[\mathrm{~kg}]} & \text { Masse } \\ \dot{m} & {\left[\mathrm{~kg} \mathrm{~s}^{-1}\right]} & \text { Massenstrom } \\ Q & {\left[\mathrm{~m}^{3} \mathrm{~s}^{-1}\right]} & \text { Volumenstrom } \\ r & {\left[\mathrm{~m}^{-1}\right.} & \text { Radius } \\ t & {[\mathrm{~s}]} & \text { Zeit } \\ v & {\left[\mathrm{~m} \mathrm{~s}^{-1}\right]} & \text { Geschwindigkeit } \\ x & {[]} & \text { Partikeldurchmesser }\end{array}$

\section{Griechische Symbole}

$\begin{array}{lll}\eta & {[\mathrm{Pa} \mathrm{s}]} & \text { dynamische Viskosität } \\ \rho & {\left[\mathrm{kg} \mathrm{m}^{-3}\right]} & \text { Dichte } \\ \tau & {[]} & \text { Durchströmungsziffer } \\ \Phi & {[\mathrm{]}]} & \text { Feststoffvolumenanteil } \\ \omega & {\left[\mathrm{s}^{-1}\right]} & \text { Winkelgeschwindigkeit }\end{array}$

\section{Indizes und Superskripte}

$\begin{array}{ll}\text { ax } & \text { axial } \\ \mathrm{f} & \text { Flüssigkeit } \\ \text { kor } & \text { korrigiert } \\ \mathrm{s} & \text { Feststoff } \\ \text { sep } & \text { Separation }\end{array}$




\section{Abkürzungen}

CFD numerische Strömungssimulationen

CSTR kontinuierlicher Rührreaktor

PFR Pfropfenstromreaktor

SQP sequential quadratic programming

\section{Literatur}

[1] J. Zhou, Adv. Manuf. 2013, 1, 1 7. DOI: https://doi.org/10.1007/ s40436 01300065

[2] M. Dosta, S. Antonyuk, E. U. Hartge, S. Heinrich, Chem. Ing. Tech. 2014, 86, 1073 1079. DOI: https://doi.org/10.1002/ cite. 201400021

[3] N. Asprion, M. Bortz, Chem. Ing. Tech. 2018, 90, 17271738. DOI: https://doi.org/10.1002/cite.201800051

[4] M. Gleiss, H. Nirschl, Chem. Eng. Technol. 2015, 38, 18731882. DOI: https://doi.org/10.1002/ceat.201500037

[5] M. Gleiss, S. Hammerich, M. Kespe, H. Nirschl, Chem. Eng. Sci. 2017, 163, 167 178. DOI: https://doi.org/10.1016/ j.ces.2017.01.046

[6] S. Wright, J. Nocedal, Numerical Optimization, Springer, New York 1999.

[7] V. Skorych, M. Dosta, E. U. Hartge, S. Heinrich, Powder Technol. 2017, 314, 665 679. DOI: https://doi.org/10.1016/j.pow tec.2017.01.061

[8] A. Erk, Rheologische Eigenschaften feindisperser Suspensionen in Filtern und Zentrifugen, Ph.D. Thesis, Universität Karlsruhe (TH) 2006

[9] M. Stieß, Mechanische Verfahrenstechnik Partikeltechnologie 1, Springer Verlag, Berlin 2008.

[10] C. Bernhardt, Klassier und Sedimentationsmethoden, Wiley VCH, Weinheim 1991.

[11] H. Nirschl, Chem. Ing. Tech. 2007, 79, 1797 1807. DOI: https:// doi.org/10.1002/cite. 200700107

[12] F. Löffler, Staubabscheiden, Thieme, Stuttgart 1988.

[13] J. Draxler, M. Siebenhofer, Verfahrenstechnik in Beispielen, Springer Vieweg, Wiesbaden 2014.

[14] J. F. Richardson, W. N. Zaki, Chem. Eng. Res. Des. 1997, 75, 82100.

[15] S. Stahl, L. E. Spelter, H. Nirschl, Chem. Eng. Technol. 2008, 31, 1577 1583. DOI: https://doi.org/10.1002/ceat.200800300

[16] T. Mladenchev, Modellierung des Filtrations und Fließverhaltens von ultrafeinen, kompressiblen, flüssigkeitsgesättigten Partikelpackungen, Ph.D Thesis, Otto von Guericke Universität Magdeburg 2007.

[17] A. Erk, H. Anlauf, W. Stahl, Chem. Ing. Tech. 2004, 76, 14941499.

[18] B. Erk, A. Luda, Chem. Ing. Tech. 2003, 75, 1250 1254. DOI: https://doi.org/10.1002/cite.200303260

[19] R. H. Müller, G. E. Hildebrand, Zetapotential und Partikelladung in der Laborpraxis, Wissenschaftliche Verlagsgesellschaft, Stuttgart 1996.

[20] M. Beiser, G. Bickert, P. Scharfer, Chem. Eng. Technol. 2004, 27, 1084 1088. DOI: https://doi.org/10.1002/ceat.200403252

[21] T. Mladenchev, J. Tomas, Chem. Ing. Tech. 2004, 76, 18141818.

[22] A. Tiselius, Kolloid Z. 1932, 59, 306309.

[23] H. Reuter, Chem. Ing. Tech. 1967, 39, 311318.

[24] W. Gösele, Chem. Ing. Tech. 1974, 46, 67.

[25] C. M. Ambler, Chem. Eng. Prog. 1952, 48, 150158.
[26] C. M. Ambler, J. Microb. Biochem. Technol. 1959, 1, 185205. DOI: https://doi.org/10.1002/jbmte.390010206

[27] C. M. Ambler, Ind. Eng. Chem. Res. 1961, 48, 430 433. DOI: https://doi.org/10.1021/ie50618a021

[28] E. B. Nauman, Ind. Eng. Chem. Res. 2008, 47, 37523766.

[29] E. B. Nauman, B. A. Buffham, Mixing in continuous flow systems, John Wiley \& Sons, New York 1983.

[30] D. Mewes, Chem. Ing. Tech. 1986, 58, 260.

[31] W. Pippel, Verweilzeitanalyse in technologischen Strömungs systemen, Akademie Verlag, Berlin 1978.

[32] M. Baerns, H. Hofmann, A. Renken, Chemische Reaktionstechnik, Wiley VCH, Weinheim 2004.

[33] B. Platzer, K. Steffani, S. Große, Chem. Ing. Tech. 1999, 71, 795807.

[34] A. Paschedag, CFD in der Verfahrenstechnik, Wiley VCH, Weinheim 2004.

[35] M. Breitling, U. Janoske, M. Piesche, Chem. Ing. Tech. 2003, 75, 184188.

[36] C. Pang, W. Tan, E. Sha, Y. Tao, L. Liu, Chem. Sci. Eng. 2012, 6, 329 338. DOI: https://doi.org/10.1007/s11705 01212055

[37] X. Romaní Fernández, H. Nirschl, Chem. Eng. Technol. 2009, 32, 719725.

[38] R. Burger, F. Concha, Int. J. Miner. Process. 1999, 55, 267282.

[39] S. Hammerich, M. Gleiß, M. Kespe, H. Nirschl, Chem. Eng. Technol. 2018, 41, 44 50. DOI: https://doi.org/10.1002/ ceat.201700104

[40] J. Ferry, S. Balachandar, Int. J. Multiphase Flow 2001, 27, 11991226.

[41] G. J. Kynch, Trans. Faraday Soc. 1952, 48, 166. DOI: https:// doi.org/10.1039/tf9524800166

[42] P. Garrido, R. Bürger, F. Concha, Int. J. Miner. Process. 2000, 60, 213 227. DOI: https://doi.org/10.1016/S0301 7516(00)00014 4

[43] R. Bürger, F. Concha, Int. J. Miner. Process. 2001, 63, 115145. DOI: https://doi.org/10.1016/S0301 7516(01)00038 2

[44] A. D. Stickland, L. R. White, P. J. Scales, AIChE J. 2006, 52, 1351 1362. DOI: https://doi.org/10.1002/aic.10746

[45] I. Takacs, G. Patry, D. Nolasco, Water Res. 1991, 25, 12631271

[46] Y. Zheng, D. M. Bagley, J. Environ. Eng. 1998, 124, 953958.

[47] B. Li, M. K. Stenstrom, Water Res. 2014, 65, 40 63. DOI: https:// doi.org/10.1016/j.watres.2014.07.007

[48] L. E. Spelter, H. Nirschl, A. D. Stickland, P. J. Scales, AIChE J. 2013, 59, 3843 3855. DOI: https://doi.org/10.1002/aic.14115

[49] W. Stahl, Fest Flüssig Trennung Band II: Industrie Zentrifugen, Maschinen und Verfahenstechnik, DRM Press, Männedorf 2004.

[50] W. Langeloh, T. Stahl, Aufbereitungstechnik 1989, 31, 331337.

[51] M. Beiser, Sedimentation submikroner Partikel in Abhängigkeit physikalisch chemischer Einflüsse und ihr Separationsverhalten in Dekantierzentrifugen, Ph.D Thesis, Universität Karlsruhe (TH) 2006.

[52] M. Gleiss, S. Hammerich, M. Kespe, H. Nirschl, Chem. Eng. Technol. 2018, 41, 19 26. DOI: https://doi.org/10.1002/ ceat. 201700113

[53] M. Konrath, Klassierung von Feinstpartikeln mittels schnell drehender Sedimentationszentrifugen, Ph.D Thesis, Karlsruher Institut für Technologie (KIT) 2016.

[54] M. Konrath, M. Hackbarth, H. Nirschl, Adv. Powder Technol. 2014, 25, 991 998. DOI: https://doi.org/10.1016/ j.apt.2014.01.022

[55] L. Spelter, Abtrennung und Klassierung kolloidaler Partikel in Zentrifugen: Experimenteller Nachweis und Modellierung der Sedimentation in halbkontinuierlichen Vollmantelzentrifugen, Ph.D. Thesis, Karlsruher Institut für Technologie (KIT) 2012. 


\section{Repository KITopen}

Dies ist ein Postprint/begutachtetes Manuskript.

Empfohlene Zitierung:

Hammerich, S.; Gleiß, M.; Nirschl, H.

Modellierung und Simulation von Vollmantelzentrifugen als ein Aspekt der voranschreitenden Digitalisierung in der Fest/Flüssig-Trennung.

2019. Chemie - Ingenieur - Technik, 91.

doi: $\underline{10.5445 / / \mathrm{R} / 1000097072}$

Zitierung der Originalveröffentlichung:

Hammerich, S.; Gleiß, M.; Nirschl, H.

Modellierung und Simulation von Vollmantelzentrifugen als ein Aspekt der voranschreitenden Digitalisierung in der Fest/Flüssig-Trennung.

2019. Chemie - Ingenieur - Technik, 91 (3), 215-227.

doi: $10.1002 /$ cite. 201800098 